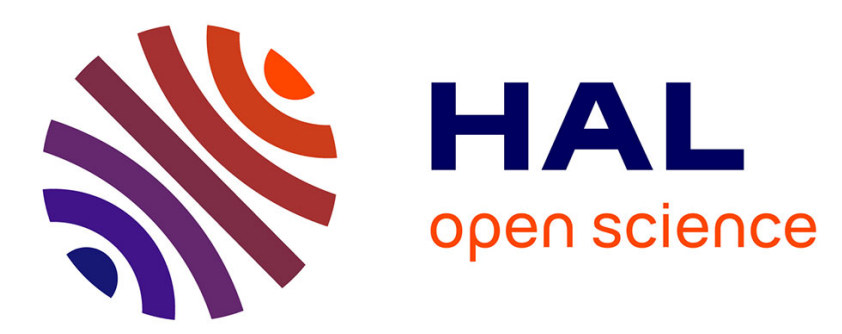

\title{
Les effets de la distance sur le discours de l'enseignant et le comportement des apprenants
}

\author{
Philippe Dessus, Pascal Marquet
}

\section{To cite this version:}

Philippe Dessus, Pascal Marquet. Les effets de la distance sur le discours de l'enseignant et le comportement des apprenants. Distances et savoirs, 2003, 1 (3), pp.337 - 360. 10.3166/ds.1.337-360 . hal-01083076

\section{HAL Id: hal-01083076 \\ https://hal.univ-grenoble-alpes.fr/hal-01083076}

Submitted on 17 Nov 2014

HAL is a multi-disciplinary open access archive for the deposit and dissemination of scientific research documents, whether they are published or not. The documents may come from teaching and research institutions in France or abroad, or from public or private research centers.
L'archive ouverte pluridisciplinaire HAL, est destinée au dépôt et à la diffusion de documents scientifiques de niveau recherche, publiés ou non, émanant des établissements d'enseignement et de recherche français ou étrangers, des laboratoires publics ou privés. 


\title{
Les effets de la distance sur le discours de l'enseignant et le comportement des apprenants
}

\author{
Philippe Dessus* — Pascal Marquet** \\ * Laboratoire des sciences de l'éducation (EA 602) \\ Université Pierre-Mendès-France \\ BP 47, F-38040 GRENOBLE CEDEX 9 \\ et IUFM, 30 av. Berthelot, F-38100 GRENOBLE \\ Philippe.Dessus@upmf-grenoble.fr_ www.upmf-grenoble.fr/sciedu/pdessus \\ ** Laboratoire des sciences de l'éducation (EA 2310), Équipe SESAM \\ Université Louis-Pasteur \\ 7, rue de l'Université F-67000 STRASBOURG \\ Pascal.Marquet@lse-ulp.u-strasbg.fr
}

RESUME : Nous réinterprétons les résultats de trois expérimentations sur l'enseignement à distance dans le cadre théorique de la distance de transaction. Selon cette notion, la perception de la distance entre un enseignant et les apprenants ne dépend pas de la distance spatiale, ou du type de média utilisé, mais plutôt de la structure du cours, les possibilités de dialogue entre enseignants et élèves, et l'autonomie de ces derniers. Nous avons étudié les effets de la distance sur le discours de l'enseignant et le comportement des étudiants en faisant les comparaisons suivantes : $1^{\circ}$ ) cours en amphithéâtre vs cours à distance; $2^{\circ}$ ) cours en amphithéâtre avec diaporama vs cours à distance avec diaporama; $3^{\circ}$ ) cours en amphithéâtre vs rediffusion de ce cours sur vidéo. Notre résultats montrent que, plus les comparaisons sont contrôlées, plus les différences entre les deux situations sont faibles.

ABSTRACT: We reanalyse here the results of three experiments on distance education within the framework of Transactional Distance, in which the participants' perception of distance do not depends on spatial distance or the use of media, but rather on: course structure, instructional dialogue and students autonomy. Three experiments are presented, in which the situational variables are more and more controlled: 1) amphitheatre lecture vs. lecture at a distance, 2) amphitheatre lecture with slides vs. lecture at a distance with slides, 3) amphitheatre lecture vs. presentation of the same videotaped lecture. We aim are highlighting the effects of distance on two main dependent variables: teacher discourse and students behaviour. Our results show that the more controlled the various situational variables, the more close the two compared teaching situations are.

MOTS-CLES : $\quad$ Enseignement à distance — Distance de transaction — Effets des médias

KEY WORDS: $\quad$ Distance learning - Transactional Distance - Media effects 


\begin{abstract}
"J'ai deux mauvaises nouvelles à propos du cours expérimental d'expression écrite en anglais diffusé par conférence assistée par ordinateur. La première est que, pour un cours d'un semestre, le groupe expérimental n'a pas progressé dans sa capacité à rédiger un essai. La seconde mauvaise nouvelle est que le groupe contrôle, utilisant des méthodes traditionnelles, n'a pas progressé non plus."
\end{abstract}

Roxanne Hiltz, conférence reportée par Ehrmann (1995)

\title{
1. Introduction
}

Il existe, dans la littérature sur les technologies éducatives, de très nombreuses références, implicites ou explicites, aux effets des médias, que ces effets soient d'ailleurs positifs ou négatifs. Sans être exhaustifs, citons en vrac les «couch potatoes», la citoyenneté par l'Internet et, plus récemment, la «fracture numérique » ou encore la cyberculture. Ces références, d'auteurs de courants théoriques très divers, développent toutes l'idée que c'est le fait d'utiliser tel ou tel média (la télévision, l'ordinateur) qui provoque en grande partie le phénomène observé (respectivement, la passivité, la citoyenneté ou la culture). Nous ne discuterons pas de leur bien-fondé ici, mais nous essaierons plutôt de répondre à la question suivante : ces effets sont-ils seulement imaginaires ou bien reposent-ils sur des résultats de recherche évaluant leur réalité?

L'enseignement à distance (EAD) occupe une place de choix dans la littérature sur les technologies éducatives et les travaux sur les usages des systèmes techniques à des fins d'EAD sont en augmentation régulière, notamment dans la communauté francophone (Desmoulins et al., 2003). Dans un tel contexte, nous pouvons déjà noter que la notion d'EAD est difficile à cerner : « enseignement » renvoie à une activité humaine mettant en œuvre des buts, une ou plusieurs méthode(s), parfois aussi du matériel. Le mot « distance » assure qu'une partie de ce matériel servira à médiatiser le contenu. L'EAD suppose donc a minima un média et une méthode d'enseignement, et c'est cette double facette qui crée des difficultés pour l'appréhender (Dringus \& Terrell, 1999), et donc pour en évaluer les effets. Nous y reviendrons plus loin.

Quoi qu'il en soit, les études autour de cette idée de l'effet positif ou négatif des technologies éducatives, qui mettent en œuvre des méthodes particulières ou qui reposent sur des médias spécifiques, peuvent schématiquement être classées en trois catégories : - l'analyse des caractéristiques du média utilisé et de ses effets ; - la comparaison de médias entre eux ; - l'analyse des processus cognitifs des utilisateurs de médias (enseignants et apprenants) réalisant une activité donnée. Nous pouvons constater que ces différents courants de recherche ne sont pas propres à l'EAD, mais sont plus généralement issus des recherches sur les médias éducatifs. À cet égard, EAD et multimédia éducatif se confondent de plus en plus, à la fois pour de nombreuses raisons techniques : les logiciels permettant de créer des cours à distance sont devenus, au fil du temps, de plus en plus aisés d'accès et de plus en plus proches de ceux conçus pour le multimédia; le raccordement massif des ordinateurs à des réseaux et/ou à l'Internet. Mais aussi pour des raisons liées à la recherche : le fait que tuteurs intelligents et systèmes d'EAD incorporent maintenant fréquemment une méthode d'enseignement, et aussi la tendance de plus en plus fréquente, dans l'EAD, qui consiste à interpréter comme favorables à ce dernier des comparaisons non significatives avec un enseignement en présence.

Cet article comprend deux parties principales. Dans la première partie, nous revenons sur les différents courants de recherche qui ont étudié les effets de l'EAD sur l'apprentissage et l'enseignement. Nous évoquons leurs paradigmes et leurs impasses respectives, mais aussi leur complémentarité. En guise d'alternative aux difficultés soulevées par ces approches, nous retenons la notion de distance de transaction (désormais DT), dont nous présentons les fondements. La deuxième partie est consacrée à la réinterprétation de trois études comparatives, que nous avons réalisées dans les termes de la DT, en nous intéressant tour à tour au discours de l'enseignant et au comportement des apprenants.

\section{Comment estimer les effets des médias sur l'apprentissage ?}

\subsection{Qu'est-ce qu'un média?}

La définition classique du média comme véhicule d'un contenu est utile dans un contexte de communication, mais plus délicate à utiliser dans un contexte d'apprentissage. En effet, pour en comprendre les effets sur l'apprentissage, il est nécessaire d'analyser plus finement le rôle de chaque composant (ou attribut, selon Clark, 1983) du média. Kozma $(1991,1994)$ a défini le média comme le triplet suivant :

- une technologie " capacités physiques, mécaniques ou électroniques d'un média, déterminant sa fonction et par extension, sa forme et d'autres caractéristiques » (Kozma, 1994, p. 11); 
- gérant un système de symboles, «ensembles d'expressions symboliques par lesquelles l'information est communiquée, à propos d'un champ de référence », par exemple la langue parlée, le texte imprimé, les partitions musicales, les cartes, etc. ;

- avec une capacité de traitement, «capacité d'un média à faire les opérations spécifiées sur les systèmes de symboles disponibles », par exemple : affichage, réception, stockage, recherche, organisation, traduction, transformation, évaluation. L'ordinateur et les logiciels autorisent de nombreux traitements : transformation de nombres en graphiques (tableur-grapheur), de voix en texte (reconnaissance vocale), d'image en texte (reconnaissance optique de caractères), etc.

Même si cette définition est opérationnelle pour étudier l'apprentissage de sujets avec des logiciels multimédia, elle ne l'est guère dans un contexte d'EAD, dans lequel, comme nous l'avons indiqué en introduction, la méthode d'enseignement est présente en tant que telle. Considérer l'EAD comme un média est trop simpliste, et fait passer à côté de nombreux effets dus à la méthode mise en place par l'enseignant. En effet, ce qu'impose l'EAD, c'est la délocalisation et la désynchronisation de la médiation éducative (Peraya, 2000), que seuls différents médias peuvent assurer, en faisant intervenir un système technique, qui lui-même contraint la démarche d'enseignement.

Mais considérer l'EAD comme un média et une méthode amène des problèmes difficilement solubles, comme le montrent, dans un autre domaine, les chercheurs ayant travaillé sur les méthodes pédagogiques (e.g., Bru, 1991). Sur quels critères peut-on se fonder pour montrer que telle ou telle méthode est mise en œuvre dans un enseignement? On retrouve dans cette question l'idée transposée de la décomposition des médias : une méthode n'est pas un objet en soi, mais un complexe dosage d'un grand nombre de variables dans le temps (Bru en répertorie une douzaine). Si l'étude des méthodes d'enseignement dans un contexte d'EAD reste donc à notre avis à faire, nous préférons ici adopter une troisième perspective : poser que l'EAD est une combinaison de média et d'une notion plus simple à définir et à observer, la distance de transaction, que nous définirons plus bas. Dans ce nouveau cadre, nous prenons en compte la spécificité de la distance, sans pour autant avoir un système à analyser qui comprenne de trop nombreuses variables indépendantes.

\subsection{La recherche sur les effets des médias}

Reprenons tout d'abord les trois lignes de recherche sur les effets des médias présentées dans l'introduction. Elles proviennent d'un travail déjà ancien, mais toujours actuel, de Salomon et Clark (1977).

Les travaux sur le choix et l'évaluation d'un média tentent de prescrire des critères d'utilisation de tel ou tel média, selon l'activité d'apprentissage voulue, sans pour autant présenter des arguments empiriques. Ils sont pour la plupart liés à un classement implicite des différents médias utilisés dans l'enseignement. Les modèles de sélection des médias entrent dans cette catégorie de travaux, que l'on peut voir présentés dans Gagné et Reiser (1982) et Romiszowski (1992). Ces modèles comportent souvent une vue plus large de la situation d'enseignement (caractéristiques des élèves, de l'enseignant, etc.), ils sont moins utilisés depuis l'introduction du multimédia informatique dans l'enseignement. Comme ils intègrent de nombreux systèmes de symboles, ils rendent moins utile et aussi plus difficile la détermination fine d'un système parmi ses concurrents.

Les travaux sur l'efficacité d'un média dans un contexte d'enseignement avec des comparaisons intermédias (voir Clark, 1994 ; Dessus \& Lemaire, 1999 ; Kozma, 1991). On se pose ici les questions « un média est-il supérieur à un autre ? et, si oui, en quoi ? ». Les nombreuses recherches expérimentales intermédia, avec groupe contrôle, entrent dans cette catégorie. L'exemple le plus célèbre de ce type de travaux est celui de Russell (2002), qui compile les résultats de comparaisons intermédia. Il s'est donné pour tâche de compiler le plus grand nombre possible d'études sur les médias - certaines ont trait à l'EAD - aboutissant à des différences non significatives entre les performances du groupe contrôle et celles du groupe expérimental. En parallèle, il existe une place pour les études menant à des différences significatives entre les résultats du groupe contrôle et ceux du groupe expérimental. Même si ce travail comporte certains biais, il permet, si le nombre de recherches aboutissant à une différence non significative continue de croître, de pouvoir montrer que le média n'a pas d'effet en lui-même sur l'apprentissage.

Les travaux sur la compréhension d'un contenu via un média utilisé pour véhiculer un contenu d'enseignement. Ces travaux, tout aussi nombreux que ceux des deux autres lignes de recherche, sont en général liés à diverses théories issues de la psychologie cognitive. En revanche, leur transposition à des problématiques d'enseignement à distance est récente (voir par exemple la transposition de la théorie de la charge cognitive à l'EAD dans van Bruggen, Kirschner \& Jochems, 2002). Ici, il est question de rendre compte de la manière dont les utilisateurs utilisent tel ou tel média en réalisant une activité donnée (écrire un texte, apprendre collaborativement, résoudre un problème, etc.). 
Ces trois lignes de recherche, si elles ont donné lieu à des travaux intéressants, comportent quelques problèmes. Tout d'abord, elles sont largement indépendantes: les travaux évaluatifs sont souvent trop prescriptifs et tiennent peu compte des résultats des recherches sur leur efficacité réelle, en situation. Les études sur l'efficacité des médias mettent rarement une théorie à l'épreuve. Enfin, les travaux sur la compréhension n'intègrent que peu souvent de groupe contrôle. Nous pensons que cette indépendance est préjudiciable à la qualité des travaux qui peuvent être menés dans chacun de ces champs. Dernier problème, il manque à ces travaux un moyen externe pour rendre compte des effets des médias étudiés. En d'autres termes, l'exposition des résultats s'énonce parfois dans les tautologies suivantes : «le média $X$ est efficace parce que nous l'avons choisi pour son efficacité », " les élèves utilisant le média $Y$ sont motivés parce qu'il est motivant d'utiliser un tel média ». Enfin, et nous nous centrons maintenant plus sur notre sujet, ces études ne sont que rarement réalisées dans un contexte d'EAD. Recensons justement ces dernières dans la section suivante.

\subsection{Les recherches sur les effets de l'EAD}

Nous nous intéressons ici aux travaux qui tentent de valider ou d'invalider l'idée d'efficacité de l'EAD, par comparaison avec d'autres contextes d'enseignement. Ce genre de comparaison est difficile à réaliser, à cause d'un problème crucial : le contrôle des variables non pertinentes pour la comparaison. En effet, comparer deux contextes suppose de ne faire varier que cet élément et de neutraliser ou de contrôler toutes les autres variables pouvant influencer les performances étudiées (Lockee, Moore, \& Burton, 2001). Cela suppose notamment de les comparer à méthodes d'enseignement égales, ce qui est une tâche difficile, comme on l'a vu plus haut. Malgré (ou à cause de) ces problèmes réels, cette question a entraîné une littérature abondante. Wisher et Olson (2001), après un examen quantitatif de la littérature visant à évaluer l'efficacité de l'EAD, montrent qu'approximativement deux études pour mille ont des caractéristiques qui permettent de les considérer comme fiables. Même si ce résultat est sans doute pessimiste, il concourt à regarder de plus près de quelle manière ces études sur l'EAD sont réalisées. Parmi les critiques les plus fréquentes à leur encontre figurent les suivantes.

La première, très fréquemment invoquée, est le manque d'informations importantes pour comprendre la manière dont l'EAD en question a été conçu. La méthode de conversion d'un éventuel cours « en présence » en cours à distance et les méthodes d'enseignement utilisées sont généralement passées sous silence. De plus, les résultats ne font que rarement apparaître des données de premier ordre comme le taux d'abandon. La seconde, tout aussi importante, concerne des biais méthodologiques (Phipps \& Merisotis, 1999). Il est très rare par exemple que les chercheurs répartissent les sujets aléatoirement dans les différents groupes, car les classes sont, dans un contexte d'EAD, pré-sélectionnées (Saba, 2000) ; de plus, parmi ces groupes figure assez rarement un groupe contrôle. En outre, la validité et la fiabilité des instruments mesurant les performances des étudiants sont peu questionnées, ce qui amène beaucoup de données concernant la satisfaction ou l'attitude des étudiants. Enfin, d'autres aspects plus généraux (Lockee, Moore, \& Burton, 2001 ; Phipps \& Merisotis, 1999) sont sources de problèmes :

— Les études sur l'EAD sont rarement inscrites dans la totalité d'un programme d'enseignement.

— Elles ne prennent que rarement en compte les différences interindividuelles des étudiants (âge, expérience, motivation, style d'apprentissage, etc.).

— Elles s'intéressent rarement aux interactions entre les multiples technologies utilisées dans l' EAD.

— Enfin, elles s'inscrivent rarement dans un cadre théorique explicite (Saba, 2000).

Les méta-analyses sont justement un moyen de synthétiser de multiples résultats, permettant une comparaison visant à rendre compte des effets de l'EAD. Il s'agit principalement de compiler les différentes comparaisons standardisées entre performances d'un groupe contrôle (apprenant en présence) et d'un groupe expérimental (apprenant à distance), dont les valeurs vont de -3 à +3 (mais un effet supérieur à 0,5 est considéré comme très grand, et un effet entre 0,2 et 0,5 est considéré comme important). Nous ne pouvons ici nous attarder trop sur les avantages et les inconvénients de ce type d'analyse (voir Kulik \& Kulik, 1989). Comme la plupart des méthodes offrant une vue synthétique d'un grand ensemble de données, la qualité des résultats obtenus est largement dépendante de la qualité de la sélection des études.

Cavanaugh (2001) a réalisé une méta-analyse des effets de l'EAD dans un niveau d'enseignement assez peu pris en compte dans ce domaine, l'enseignement primaire, en compilant les résultats de 19 études expérimentales. Elle montre que l'effet moyen est très bas $(0,147$, écart type 0,48$)$, ce qui ne permet pas de rejeter l'hypothèse nulle de l'équivalence de l'EAD et de l'enseignement « traditionnel » en primaire. Toutefois, lorsqu'on supprime les études ayant pour contenu les langues secondes, l'effet passe à 0,344 . Il est également à noter qu'aucune des variables recensées dans cette méta-analyse n'a d'effet significatif sur la taille de l'effet. Une autre méta-analyse récente (Machtmes \& Asher, 2000), compile les résultats de 19 études expérimentales sur l'EAD, au niveau universitaire, cette fois. L'effet moyen de ces études est quasi nul $(-0,0093)$. Toutefois, une analyse plus précise montre que trois variables influencent significativement l'efficacité des performances des 
étudiants. Il s'agit du type de communication: une communication synchrone (two-way interaction) est significativement plus efficace qu'une communication asynchrone; du lieu dans lequel est délivré l'enseignement (le lieu de travail offre de meilleures performances que la maison); et, enfin, de la matière enseignée, comme dans l'étude précédente. En revanche, toutes les autres caractéristiques organisationnelles (les caractéristiques du matériel utilisé : vidéo, polycopiés; le fait que l'organisateur du cours à distance soit disponible ou bien connaisse le sujet enseigné, etc.) n'ont pas d'effet significatif sur les performances des étudiants.

Les résultats de ces deux méta-analyses vont plutôt dans le même sens : un impact restreint de l'utilisation de l'EAD. En effet, le contenu est un des facteurs influençant l'efficacité des dispositifs, et les facteurs matériels ont peu d'influence. Toutefois, il faut considérer avec méfiance les effets causés par la technologie. Attribuer à la technologie elle-même des effets, comme le fait l'étude de Machtmes et Asher (2000), c'est oublier que l'étude ne dit pas comment les sujets utilisent réellement cette technologie. Disposer d'une possibilité de communication synchrone, ce n'est pas nécessairement l'utiliser comme telle, d'autant que des problèmes techniques (pannes, brouillages, etc.) dégradent souvent la communication (voir par exemple Périn \& Gensolen, 1992).

Il nous faut maintenant discuter cet effet non significatif de l'EAD. De nombreux auteurs (dont les auteurs des deux méta-analyses précédentes) l'interprètent comme un résultat positif: l'EAD est aussi efficace que l'enseignement en présence. Or, comme le signalent Lockee, Burton et Cross (1999), de nombreux résultats de recherches ne montrant aucune différence significative entre un groupe d'étudiants à distance et un groupe en présence sont utilisés pour conclure que l'EAD permet des performances aussi bonnes que celles en présence. Le problème est que rien ne permet de les utiliser ainsi. En effet, les tests statistiques utilisés ne sont pas conçus pour montrer une supériorité d'un groupe sur l'autre, mais le rejet éventuel d'une égalité (hypothèse nulle). Pour Lockee et ses collègues, cela revient à confondre, en droit, le jugement «non coupable » avec le jugement « innocent». Conclure à la non-culpabilité d'une personne, c'est juger que les éléments du dossier (i.e., les variables dépendantes) ne sont pas suffisants pour établir la culpabilité de cette dernière. Mais cela ne veut pas pour autant dire qu'elle est innocente.

De même, établir que la comparaison entre deux contextes d'enseignement (dont un « traditionnel ») aboutit à un résultat non significatif, cela signifie, non pas qu'ils sont également performants, mais qu'on n'a pas pu établir de différences, avec les moyens de mesure employés. Par ailleurs, ces moyens sont supposés se référer à des hypothèses, qui elles-mêmes renvoient à des modèles. Le fait que des différences n'aient pu être établies invalide vraisemblablement les hypothèses émises et remet partiellement en cause le modèle choisi. Or, tant que le modèle ne rend pas explicitement compte des effets de l'EAD ou de l'un ou l'autre de ses déterminants, la différence non significative n'est pas une preuve d'égalité de résultats. Sans sombrer dans le pessimisme de Wisher et Olson (2001) que nous évoquions plus haut, il paraît hasardeux d'utiliser ce type de résultat pour conclure à l'intérêt de l'EAD, au sens où il serait également «performant» que l'enseignement dispensé en présence.

Il ressort, des résultats des différentes lignes de recherche que nous venons de décrire, l'idée qu'il faut se centrer sur une autre problématique que celle strictement comparatiste. Nous avons tout d'abord montré la difficulté de caractériser et de contrôler avec suffisamment de précision la méthode d'enseignement utilisée dans un EAD. Nous avons montré la difficulté de mettre au jour des différences intermédias, ainsi que celle, non moins grande, de sélectionner des variables dépendantes qui permettraient l'expression de telles différences. Plus précisément, il nous semble plus intéressant de montrer, non pas que l'EAD apporte un «plus » par rapport à l'enseignement en présence, mais plutôt de montrer en quoi le fait de faire (ou de suivre) un cours à distance peut avoir une influence sur certaines caractéristiques du cours ou du comportement des étudiants. La solution que nous avons choisie pour surmonter ces difficultés consiste à considérer l'EAD comme la coexistence d'un média et des conséquences du recours à ce média, à travers la notion de distance de transaction. Comme nous allons le voir dans la section suivante, cette notion se veut à la fois opérationnelle et prédictive de certains effets de l'EAD.

\subsection{La notion de distance de transaction}

Moore (1993), l'auteur de cette notion (pour des revues en français, voir Bouchard, 2000 ; Dessus et al., 1997), ne cherche pas à montrer qu'un média (dans un contexte d'enseignement en présence ou à distance) est plus approprié qu'un autre dans telle ou telle circonstance, mais tente plutôt de rendre compte de ses effets sur l'enseignant et l'apprenant. Deux paramètres la déterminent directement (cf. figure 1) - la structure du cours : « La structure rend compte de la rigidité ou de la flexibilité des objectifs éducatifs du programme, des stratégies d'enseignement et des méthodes d'évaluation» (id., p. 26) (i.e., ses possibilités d'adaptation aux élèves, la 
quantité d'informations optionnelles pouvant être fournies aux étudiants, le niveau du contenu délivré); - le dialogue (i.e., la richesse et la fréquence des interactions entre enseignants et élèves, ou interélèves). La DT d'une situation d'enseignement est d'autant plus grande qu'on minimise le dialogue tout en rigidifiant la structure du cours, et vice versa.

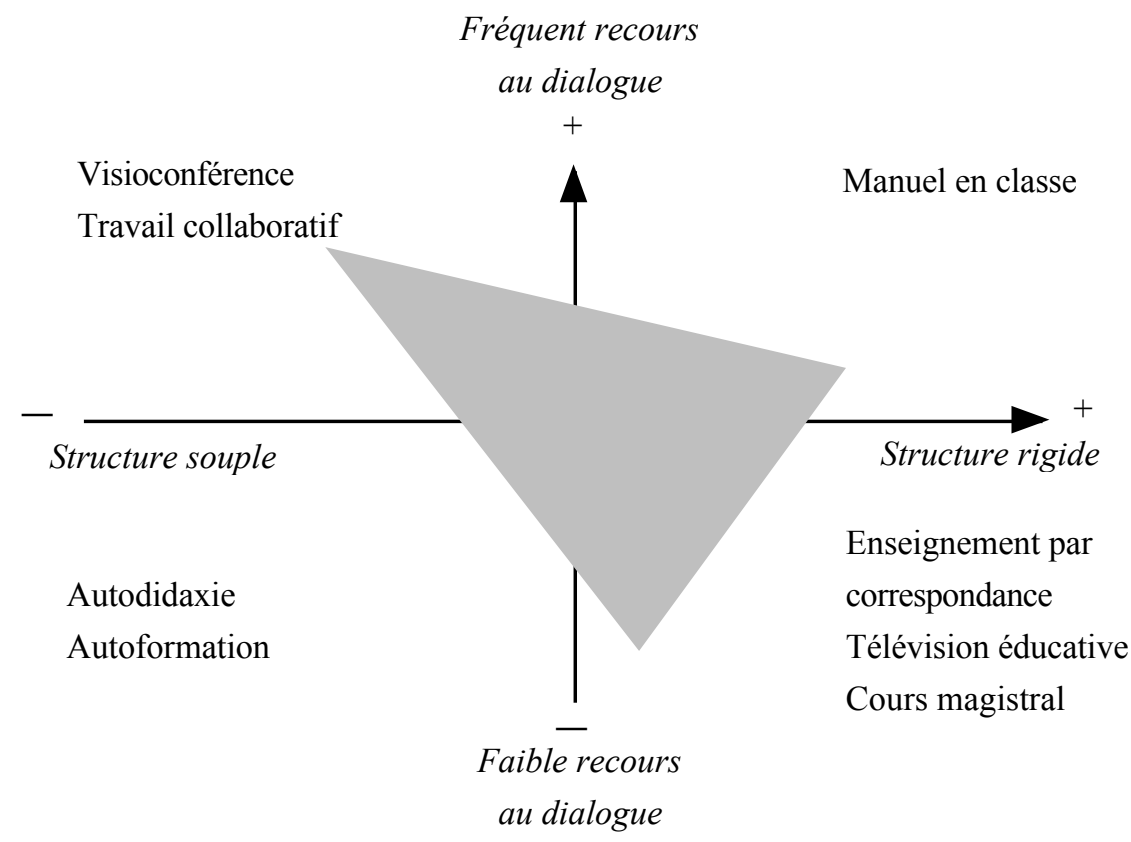

Figure 1. La distance de transaction, d'après Moore (1993), reproductions partielles de Dessus \& al. (1997, p. 141) et Bouchard (2000, p. 70). L'épaisseur de la flèche représente la grandeur de la distance de transaction.

À ces deux paramètres s'ajoutent un troisième, indirect, l'autonomie des étudiants, c'est-à-dire le fait que ce sont ces derniers, plutôt que l'enseignant, qui déterminent les buts, les expériences d'apprentissage, ou encore les décisions d'évaluation du programme d'apprentissage. Cette autonomie, en règle générale, crôt avec la DT. Plus précisément, Moore suppose que cette autonomie est d'autant plus grande que la structure et le dialogue sont faibles, et vice versa. Il apparaît donc que la DT sert bien à distinguer les différents dispositifs d'enseignement plutôt qu'à effectuer des mesures.

Cette notion est intéressante en ce qu'elle permet d'opérationnaliser la perception de distance par les étudiants et l'enseignant dans des situations d'enseignement. Plus simple que la notion de méthode d'enseignement, elle en est un sous-ensemble, sans faire intervenir des paramètres difficiles à évaluer, comme les finalités ou les objectifs d'un enseignement. Elle peut être aussi bien caractérisée dans des contextes d'enseignement en présence ou à distance, ce qui autorise les comparaisons que nous présenterons plus loin. Toutefois, elle a été critiquée pour son manque de précision et la difficulté de pouvoir la tester (Garrison, 2000) :

- les exemples donnés par Moore ne concernent que les effets de médias (matériel), et non de situations. Or, principalement, la flexibilité des objectifs et méthodes dépendent de l'enseignant et ne sont que faiblement contraints par les médias eux-mêmes ;

- il est difficile de savoir, à lire son auteur, si la structure et le dialogue se distribuent sur un continuum, ou bien sont des variables discontinues (i.e., forment un tableau croisé) ;

- la variable «structure» est difficile à déterminer quantitativement: les exemples donnés par Moore de programmes très structurés (i.e., programme diffusé par vidéocassette) prêtent à discussion, car il semblent plutôt être des exemples de cas où la variable « dialogue » est inexistante ;

— enfin, cette même variable «structure » est difficile à mesurer dans l'absolu : elle ne peut se mesurer qu'en comparant deux types de discours d'enseignant pendant son cours. Des différences entre ces deux types montreraient un cours peu structuré, alors que de faibles différences montreraient un cours très structuré.

Malgré quelques tentatives (Chen, 1998 ; Jung, 2001 ; Saba \& Shearer, 1994), il existe peu de validations empiriques de cette notion. La raison en est sans doute la difficulté de mesurer empiriquement cette distance, qui 
est la plupart du temps issue des avis des étudiants. Par exemple, l'étude de Chen (1998) a évalué, à partir de questionnaires d'étudiants à distance, le niveau perçu de dialogue, autonomie, et DT dans les cours. Cette étude ne valide que très partiellement l'hypothèse de Moore : la DT n'est pas affectée directement par la structure ni par l'autonomie des étudiants, mais elle décroît quand les discussions intraclasse augmentent. Toutefois, la DT est inversement proportionnelle aux résultats des étudiants.

Si la notion de DT offre de concevoir l'EAD autrement que comme un simple média ou une simple méthode, ou même simplement les deux, sa mise à l'épreuve dans des contextes de formation soulève quelques difficultés. Dans la partie qui suit, nous allons essayer de montrer que trois de nos travaux, tout en s'inscrivant dans les trois lignes de recherches ci-dessus, peuvent aussi être présentés à travers le prisme de la DT. Ces travaux s'inscrivent naturellement dans une théorie du champ de la recherche sur l'enseignement à distance, et permettent de manipuler des caractéristiques de médias relativement fines (présentation de diaporamas, vidéo), pour déboucher sur la prise en compte de divers processus cognitifs des apprenants. Mais leur principal intérêt est qu'ils valident la notion de DT dans son statut de conception alternative des effets de l'EAD, tout en révélant aussi les limites, que d'autres travaux devront confirmer.

\section{3. Étude des effets de la distance sur la structure du cours et l'autonomie des apprenants}

\subsection{Trois comparaisons entre des situations en présence et à distance}

Nous avons vu plus haut que les comparaisons intermédias ne rendent pas l'EAD différent des dispositifs d'enseignement classiques, ce qui prête à confusion. L'intérêt de la notion de DT réside dans la vision qu'elle donne de l'EAD, qui n'est plus considéré comme plus ou moins efficace qu'un autre type d'enseignement, mais comme une situation qui combine des formes particulières de dialogue et de structure et qui favorise une autonomie plus ou moins importante des apprenants.

$\mathrm{Au}$ cours de ces dernières années, nous avons conduit plusieurs études, consistant à comparer différents discours pédagogiques selon qu'ils sont produits dans des conditions d'enseignement magistral ou qu'ils sont mis à distance par différents médias (téléprésentation, vidéogramme, etc.), et à comparer le comportement d'auditeur des apprenants selon que l'enseignant est présent ou non. Nous nous proposons de mettre à l'épreuve la notion de DT, non pas au travers de déclarations d'apprenants, mais au moyen d'observations quantitatives qui portent sur le contenu de l'enseignement délivré et sur le comportement d'auditeur des apprenants.

Le contenu permet en effet d'appréhender la structure si difficile à mesurer a priori sous l'hypothèse que le contenu d'un cours sera d'autant plus structuré que le discours prononcé en présence et le discours prononcé à distance apparaîtront semblable. Cela signifie que, lorsqu'un enseignant réalise de multiples versions d'un cours, on peut penser que ce cours a une structure d'autant plus rigide que ces multiples versions sont semblables. Le comportement des apprenants, quant à lui, permet d'approcher l'autonomie sous l'hypothèse que la variabilité du comportement des apprenants est d'autant plus grande que l'est aussi l'autonomie.

Les études que nous reprenons (Lemaire, Dessus, \& Baillé, 1998 ; Lemaire, Marquet, \& Baillé, 1997 ; Marquet \& Herzog, 1999) mettent en jeu quatre situations d'enseignement, et trois comparaisons deux à deux de ces dernières :

1. un enseignement en amphithéâtre « classique », dans lequel l'enseignant expose un cours en présence de ses étudiants ;

2. le même type d'enseignement que précédemment, mais appuyé par un diaporama ;

3. un enseignement à distance en audioconférence synchrone, dans lequel l'enseignant expose un cours à des étudiants à distance, appuyé par un diaporama ;

4. un enseignement à distance, diffusé en asynchrone. Il consiste en la rediffusion par vidéoprojecteur d'un cours en amphithéâtre « classique », sans diaporama (un cours selon la situation 1).

Chacune de ces situations présente l'intérêt d'illustrer certaines des variables de la DT, comme indiqué dans le tableau 1 ci-dessous. La comparaison entre certaines de ces situations, à discipline équivalente, permet donc de voir quels types de variations des discours de l'enseignant et de comportement des apprenants génèrent des situations supposées correspondre à des distances de transaction différentes. Les comparaisons suivantes vont être faites :

1. Situation 1 vs situation 3 : c'est la comparaison de deux situations écologiques, en ce qu'elles sont chacune les plus représentatives de deux types d'enseignement : les enseignements universitaires en présence ne sont pas encore systématiquement appuyés de diaporamas, alors que c'est le cas de la grande majorité de ceux à 
distance. Deux variables seront étudiées : le discours de l'enseignant et le comportement des apprenants. Leur comparaison fait intervenir des situations différentes des points de vue de la structure du cours, du dialogue et de l'autonomie (voir Tableau 1), ce qui rend impossible la détermination de l'effet de la distance. Cela nous a donc amenés à réaliser deux autres comparaisons, plus contrôlées.

2. Situation $2 v s$ situation 3 : ici, l'enseignant s'appuie, dans les deux cas, sur un diaporama. Pour des raisons techniques, seul le discours de l'enseignant sera étudié dans cette deuxième comparaison. Cette nouvelle comparaison nous permet de vérifier l'éventuel effet du dialogue, puisque les deux autres paramètres de la DT sont jugés équivalents (voir Tableau 1). Toutefois, dans cette deuxième comparaison, il est difficile d'évaluer correctement l'effet de la distance sur le comportement des apprenants, car le contenu du discours de l'enseignant peut sensiblement varier d'une situation à l'autre. C'est l'objet de la dernière comparaison.

3. Situation 1 vs situation 4 : ici, le discours de l'enseignant, provenant de la situation 1 et diffusé par enregistrement dans la situation 4, est rigoureusement identique. Il est donc possible d'observer l'effet de la distance sur l'autonomie des apprenants via leur comportement. Il faut noter ici que les niveaux de structure du cours et de dialogue, par construction strictement identiques à ceux de la situation 1 puisque figés par vidéo, sont à distinguer des niveaux de structure et de dialogues réels, vécus par les apprenants de la situation 4. Nous les avons reportés entre parenthèses dans le tableau 1 ci-dessous.

Le tableau 1 ci-dessous montre que, de la situation 1 à la situation 4, la structure du cours est de plus en plus contrainte, et que les possibilités de dialogue diminuent; l'autonomie des apprenants, elle, va en augmentant. Passons maintenant à l'exposé des différentes études.

Tableau 1. Modalités des déterminants de la DT, et contenu enseigné selon les situations observées. Entre parenthèses, niveaux de structure et de dialogue réels des apprenants, puisque la situation 4 est la diffusion d'un film de cours

\begin{tabular}{|c|c|c|c|c|}
\hline Situation & Contenu & Structure & Dialogue & Autonomie \\
\hline 1. Cours amphi présence & $\begin{array}{l}\text { Sciences économiques, } \\
\text { sciences de l'éducation }\end{array}$ & - & + & - \\
\hline $\begin{array}{l}\text { 2. Cours amphi présence et } \\
\text { diaporama }\end{array}$ & Sciences économiques & + & + & $=$ \\
\hline $\begin{array}{l}\text { 3. Cours amphi distance } \\
\text { audioconférence }\end{array}$ & Sciences économiques & + & - & $=$ \\
\hline 4. Cours filmé asynchrone & Sciences de l'éducation & $-(++)$ & $+(--)$ & + \\
\hline
\end{tabular}

\subsection{Comparaison écologique: amphithéâtre vs audioconférence (effets sur le discours et sur le comportement)}

À l'occasion de cette première étude (Lemaire, Marquet, \& Baillé, 1997), nous avons comparé deux situations «écologiques» et leurs effets respectifs sur le discours de l'enseignant et le comportement des étudiants. Notre problématique a été d'étudier les transformations subies par un cours de comptabilité de DEUG de sciences économiques, selon qu'il est dispensé dans un amphithéâtre ou en téléprésentation. En amphithéâtre, le cours était suivi par une centaine d'étudiants à raison de séances de trois heures. La téléprésentation, consistait en la retransmission, en direct sur un site distant, de la voix de l'enseignant, appuyée par des transparents, à raison de deux heures par séance ; un moniteur sur place était chargé de donner la parole aux étudiants voulant intervenir sur le déroulement du cours. Comme nous l'avons indiqué dans le tableau 1 supra, les deux situations se distinguent à la fois par la structure, plus rigide en téléprésentation, ainsi que par l'autonomie des apprenants, plus grande à distance.

Le comportement de l'enseignant et des étudiants a été enregistré lors d'un cours dans chaque situation. La méthode d'analyse du discours visait à rechercher d'éventuelles différences de structuration du discours de l'enseignant, en décomptant quatre unités morphosyntaxiques issues de Bronckart (1985, voir Encadré 1 suivant), sur deux extraits de dix minutes.

Encadré 1. Une présentation de la méthode de Bronckart (1985)

L'intérêt de cette approche d'analyse du discours réside dans la mise en relation entre des unités morphosyntaxiques apparaissant dans des textes et leurs conditions de production. Dans un cadre théorique de type développemental, l'auteur 
identifie trois grandes familles d'opérations langagières à l'œuvre dans l'activité discursive : la contextualisation, la structuration et la textualisation. La contextualisation regroupe des opérations d'un premier niveau, préalable à la production langagière. Il s'agit essentiellement de processus de représentation et de choix de valeurs des paramètres de l'extralangage, entendu ici comme tout ce qui est extérieur à la langue. À un second niveau, la structuration comprend les opérations qui déterminent la trame du texte, son enveloppe linguistique. Les conditions d'interactions sociales orientent, par le jeu de ces opérations, les plans discursifs du locuteur. Enfin, à un troisième niveau, les opérations de textualisation concourent à la mise en texte et à l'organisation séquentielle des unités verbales, organisation qui subit l'influence des deux types d'opérations précédents. Ce sont donc ces trois niveaux d'opérations, croisées avec la situation de production, qui vont conduire le locuteur à choisir tel ou tel auxiliaire modal, telle ou telle désinence verbale, tel ou tel organisateur argumentatif.

À la lumière de la notion de DT, on peut raisonnablement penser qu'en situation de téléprésentation, l'importante activité préalable de l'enseignant, consistant notamment à réaliser des transparents, devrait se refléter par un discours plus structuré. Cette hypothèse nous conduit à retenir et à dénombrer les indicateurs de structuration suivants :

1. le nombre d'auxiliaires de mode (vouloir, devoir, falloir), révélateurs de l'action de l'énonciateur sur l'interlocuteur ;

2. le nombre de phrases non déclaratives (interrogatives directes, impératives et exclamatives), pour les mêmes raisons ;

3. le nombre d'organisateurs argumentatifs lexico-syntaxiques (adverbes, conjonctions), structurant l'exposé en établissant des jalons textuels ;

4. le nombre d'anaphores pronominales, qui participent à la cohésion du texte.

Les deux premiers indicateurs devraient se retrouver en plus grand nombre dans le discours prononcé en amphithéâtre et les deux derniers devraient apparaître plus fréquemment dans le discours prononcé en téléprésentation. La comparaison entre les deux situations va bien dans le sens attendu ( $c f$. Tableau 2). Les occurrences des indices retenus se révèlent significativement différentes selon le type de discours $\left(\chi^{2}=16,24 ; p\right.$ $<.01$ à ddl = 3). Il se confirme donc que le cours en téléprésentation repose sur un discours nettement plus structuré que le même cours prononcé en amphithéâtre.

Tableau 2. Nombre d'unités linguistiques dans les deux types de discours (d'après Bronckart, 1985). La présence de nombres décimaux provient d'un réajustement au même nombre de mots (1 000)

\begin{tabular}{lcc}
\hline Unités linguistiques (différence attendue) & Amphithéâtre & Téléprésentation \\
\hline 1. Auxiliaires de mode $(>)$ & 13 & 5,8 \\
2. Phrases non déclaratives $(>)$ & 12 & 4,7 \\
3. Org. argumentatifs lexico-syntaxiques $(<)$ & 7 & 19 \\
4. Anaphores pronominales $(<)$ & 11 & 26 \\
\hline
\end{tabular}

En complément à l'analyse du discours de l'enseignant, nous avons observé le comportement d'auditeur au cours des mêmes séquences de dix minutes de seize apprenants (huit pour chaque situation), en nous basant sur le décompte des durées consacrées à quatre activités observables : la prise de notes, l'écoute, l'observation des supports d'information (textes et schémas inscrits au tableau, transparents), le décrochage momentané. Certains regroupements de ces activités nous renseignent aussi, de manière très générale encore, sur les activités mentales susceptibles d'être imposées aux apprenants par chaque situation. Une première distinction entre prise d'informations et restitution d'informations est possible en considérant les activités d'écoute et d'observation comme relevant d'une activité de prise d'informations et l'activité de prise de notes comme procédant d'une activité de restitution d'informations ( $c f$. Tableau 3 ci-dessous).

Ces comportements ont la propriété d'être distincts les uns des autres et, lorsqu'ils sont réunis, de constituer un bon indicateur de l'activité de suivi d'un cours. Ils sont en outre très caractéristiques et donc distinguables sans ambiguïté à partir d'enregistrements vidéoscopiques. Par précaution, nous avons fait visionner les enregistrements par deux juges et nous avons considéré que le sujet observé était engagé dans telle ou telle activité uniquement lorsque les deux observateurs étaient en accord. En ce qui concerne les comportements isolés, la différence d'autonomie dont nous cherchons à rendre compte ici devrait se manifester par des différences de durées d'observation des supports et de durées de décrochage, sans que nous ne puissions prédire, comme nous l'avons fait pour le discours, dans quelle situation l'une ou l'autre des activités devrait occuper le plus de temps. Pour ce qui est des regroupements de comportements, seule une éventuelle différence de durée de 
prise d'informations nous paraît informante : elle témoigne, selon nous, de la liberté laissée à l'apprenant de tirer ou non parti de l'information délivrée par l'enseignant.

Tableau 3. Durées moyennes en secondes (sur un total observé de 600) des comportements des apprenants en situations d'enseignement classique et de télé présentation

\begin{tabular}{|c|c|c|c|c|c|c|}
\hline \multirow[b]{2}{*}{ Situations } & \multicolumn{6}{|c|}{ Comportements des apprenants (durée en s) } \\
\hline & $\begin{array}{c}\text { Prise de } \\
\text { notes }\end{array}$ & Écoute & Observ. & Décroch. & $\begin{array}{l}\text { Prise } \\
\text { d'info. }\end{array}$ & $\begin{array}{c}\text { Restitution } \\
\text { d'info. }\end{array}$ \\
\hline Amphi & 267,6 & 74,1 & 198,5 & 91,6 & 272,6 & 267,6 \\
\hline Téléprés. & 203,2 & 312,9 & 92,0 & 26,9 & 404,9 & 203,2 \\
\hline
\end{tabular}

Les durées consacrées à trois des quatre activités observées chez les apprenants diffèrent significativement selon la situation d'enseignement ( $c f$. Tableau 3 ci-dessus). L'écoute occupe plus de temps en téléprésentation qu'en amphithéâtre $(U(0,64)=3,34 ; p<.01)$, tandis que l'observation des supports et les décrochages sont plus longs en amphithéâtre que pendant la téléprésentation (respectivement, $U(19,45)=2,1 ; p<.05$ et $U(12,52)=$ $2,42 ; p<.05)$. La prise de note, quant à elle, ne semble pas évoluer de façon significative $(U(19,45)=1,37$; n.s.). Par ailleurs, la durée de prise d'informations apparaît plus importante pour la téléprésentation que pour le cours magistral $(U(12,52)=2,1 ; p<.05)$. La différence de durée de restitution d'informations, quant à elle, n'est pas significative $(U(19,45)=1,37 ;$ n.s. $)$.

Il ressort de cette première observation qu'il y a bien une variabilité de structure du discours de l'enseignant et de l'autonomie des apprenants selon que l'enseignement est dispensé en présence ou à distance. Il reste que le manque de contrôle des situations observées nuit à l'interprétation des résultats : on ne peut déterminer avec certitude ce qui peut être la cause des différences. Toutefois, comme nous l'avons souligné plus haut (voir $\S 2.3$ ), de tels résultats significatifs ne sont pas si fréquemment mis au jour dans la littérature. S'il était intéressant de bénéficier de conditions très proches de la réalité dans un premier temps, l'analyse de situations suivant la DT nécessite une plus grande rigueur. Les deux observations qui suivent vont nous permettre d'y parvenir, d'abord en faisant en sorte que les discours prononcés soient plus proches (même contenu en amphithéâtre vs. en audioconférence), puis rigoureusement identiques (même cours en amphithéâtre vs. en vidéodifféré). Voyons si ces différences s'accroissent ou s'estompent lorsque que le contrôle des variables indépendantes augmente.

\subsection{Comparaison contrôlée 1 : amphithéâtre avec diaporama vs audioconférence (effets sur le discours)}

Il s'agit ici de supprimer la variation des possibilités de structure du cours et de ne conserver que la variation du dialogue, tout en la contrôlant expérimentalement. Ainsi, les deux situations que nous avons comparées dans cette deuxième étude se caractérisent par le fait que les apprenants sont confrontés au même type de dispositif: un diaporama; et que le contenu du cours diffusé est identique. Le principal sujet d'observation est ici le discours de l'enseignant, analysé selon deux méthodes différentes: la méthode de Bronckart, comme précédemment, ainsi qu'une méthode d'analyse statistique proche d'une analyse factorielle, l'Analyse de la sémantique latente (désormais LSA pour Latent Semantic Analysis, Landauer \& Dumais, 1997).

Le cours observé est un cours de comptabilité nationale de première année de DEUG de Sciences économiques, dispensé de deux façons, de manière à ce que la seule variable susceptible d'avoir un effet sur le discours de l'enseignant soit la distance : - en amphithéâtre, sous forme de cours magistraux accompagnés de transparents, suivis par 109 étudiants ; - par un cours à distance (audioconférence), en direct, appuyé par les mêmes transparents. Ce cours est suivi par 57 étudiants. L'enseignant n'est pas visible par les étudiants. Un assistant, sur place, est chargé de donner la parole aux étudiants (voir Lemaire, Dessus, \& Baillé, 1998, pour un exposé complet de l'expérimentation). La première analyse du discours reprend donc la méthode de Bronckart, décrite supra, également sur deux extraits des discours. Contrairement à l'étude précédente, le résultat du test statistique ne montre aucune différence significative $\left(\chi^{2}=0,99 ;\right.$ n.s. à ddl $\left.=3\right)$ entre ces deux types de discours ( $c f$. Tableau 4). 
Tableau 4. Nombre d'unités linguistiques dans les deux types de discours (d'après Bronckart, 1985). La présence de nombres décimaux provient d'un réajustement au même nombre de mots (1 000)

\begin{tabular}{lcc}
\hline Unités linguistiques (différence attendue) & Amphithéâtre & Téléprésentation \\
\hline 1. Auxiliaires de mode $(>)$ & 3 & 2,4 \\
2. Phrases non déclaratives $(>)$ & 9,4 & 4,8 \\
3. Org. argumentatifs lexico-syntaxiques $(<)$ & 30,1 & 26,9 \\
4. Anaphores pronominales $(<)$ & 41,4 & 29,1 \\
\hline
\end{tabular}

Il est intéressant de noter, que dans des conditions de structure de cours semblables, les possibilités différentes de dialogue ne se répercutent pas sur les indicateurs ci-dessus. Toutefois, compte tenu de la faible durée de l'extrait analysé et du nombre restreint d'indicateurs sur lesquels reposent l'analyse, nous avons souhaité approfondir cette absence de variation. La deuxième analyse (Dessus, soumis à publication) a alors consisté à traiter le corpus entier des deux discours avec LSA (voir Encadré 2). Ces derniers ont été segmentés par diapositive et traité par LSA, conjointement à un corpus de 10 millions de caractères provenant du Monde, corpus qui permet d'affiner la connaissance de la langue. Ensuite, LSA détermine la proximité sémantique entre le discours de l'enseignant pendant l'exposition d'une diapositive en présence et le discours à propos de la même diapositive à distance (valeurs comprises entre -1 et 1 ). Cela nous permet de calculer la proximité moyenne entre les séquences en présence et celles à distance : 0,80 (écart type 0,08 ). Cette valeur, très élevée si on la compare à d'autres obtenues dans la littérature (voir par exemple Wolfe et al., 1998), est très supérieure à la valeur moyenne de la proximité de deux diapositives en présence $(0,06)$ ou à distance $(0,07)$. Il y a donc, d'un point de vue sémantique, une différence très faible entre les deux types de discours.

Pour autant, le fait qu'aucune différence significative entre les deux situations n'ait été relevée à l'issue des deux analyses ne nous autorise bien sûr toujours pas à conclure que ces situations sont les mêmes. La seule chose que nous pouvons dire, c'est que le fait de produire un cours appuyé par un diaporama en présence ou à distance, n'a pas d'effet sur la structure perceptible par les moyens d'investigation choisis.

Encadré 2. Une présentation de l'analyse de la sémantique latente

L'Analyse de la sémantique latente (LSA, pour Latent Semantic Analysis) commence à être utilisée pour rendre compte du contenu des discours ou textes dans certains systèmes d'enseignement à distance (Teplovs \& van Aalst, 1998). Le principe de LSA est le suivant : le sens d'un mot est défini statistiquement à partir de l'ensemble des contextes (i.e., paragraphes, phrases, textes) dans lesquels ce mot apparaît. Par exemple, le mot avion va apparaître souvent conjointement à des mots comme décoller, aile, aéroport et très peu fréquemment à des mots comme pédagogie ou dinosaure. Cependant, cette information statistique sur le contexte d'un mot n'est pas suffisante pour en définir le sens, puisqu'elle ne dit rien quant aux liens sémantiques avec tous les autres mots n'apparaissant jamais conjointement à ce mot. Il faut pour cela un mécanisme permettant de croiser les informations de co-occurrence propres à chaque mot. Ensuite, les différents mots du corpus sont projetés dans un espace d'environ 300 dimensions, ce qui autorise leur comparaison et, par sommation des vecteurs des mots, la comparaison de paragraphes. De très nombreux résultats expérimentaux montrent que LSA est un modèle cognitif acceptable de la représentation et l'acquisition de connaissances à partir de textes (Lemaire \& Dessus, 2003).

\subsection{Comparaison contrôlée 2 : amphithéâtre vs amphithéâtre vidéodifféré (effets sur le comportement des étudiants)}

À l'inverse de la précédente observation, nous supprimons les variations de structure du cours et dialogue, pour nous concentrer sur celle de l'autonomie des apprenants. Nous affinons nos observations du comportement d'auditeur des apprenants, puisqu'il s'agit du même discours prononcé dans les deux conditions suivantes : un cours de docimologie de licence de sciences de l'éducation en amphithéâtre ; - la projection sur grand écran en amphithéâtre, en présence de l'enseignant en cas d'éventuelles questions, de l'enregistrement vidéo de ce cours.

L'analyse du comportement des apprenants a porté sur les durées des quatre mêmes activités que dans la première comparaison. Compte tenu de ce que nous avons vu plus haut, nous nous attendons à ce que l'activité d'écoute occupe moins de temps en amphithéâtre qu'en vidéodifféré et, qu'inversement, l'activité d'observation accapare les apprenants moins longtemps en vidéodifféré qu'en amphithéâtre. Enfin, la prise d'informations devrait, quant à elle être plus importante en vidéodifféré qu'en amphithéâtre, l'autonomie des apprenants étant 
supposée croître en même temps que la DT. Seize sujets, à raison de huit pour chaque situation et choisis sur des critères de qualité d'image, ont été observés de la $15^{\mathrm{e}}$ à la $25^{\mathrm{e}}$ minute d'un cours de deux heures, à partir de l'enregistrement vidéoscopique de l'auditoire. Nous avons en outre recueilli par interview les impressions des étudiants de la deuxième situation en fin de séance.

L'analyse des interviews indique que deux types d'éléments concourent à rendre le cours vidéodifféré difficile à suivre, les privant ainsi d'autonomie. Un premier ensemble de remarques laisse entendre que la diffusion d'un cours magistral sur écran place les étudiants sur un registre comportemental plus près de celui du téléspectateur que de l'apprenant. De ce fait, la position externe que confère la situation aurait tendance à faciliter le décrochage de l'étudiant, et c'est précisément l'effort pour ne pas décrocher qui paraît coûteux. Cette impression n'est pas confirmée par l'observation du comportement, comme nous le verrons plus bas. Par ailleurs, l'absence de régulation du débit de parole en fonction de la réceptivité des étudiants contribue encore à augmenter la difficulté à suivre. Un deuxième ensemble de remarques suggère que ce que les étudiants voient n'est pas toujours ce dont ils ont besoin. En effet, dans une situation classique, l'étudiant choisit d'écouter l'enseignant, de regarder le tableau, de prendre des notes ou de faire deux choses en même temps, ou même de ne rien faire. Sur l'enregistrement, ce choix est déjà fait pour lui et les éléments qui sont projetés ne correspondent pas toujours à ce dont tel ou tel sujet a besoin. Par exemple, certains regrettent de ne pas pouvoir revenir sur le tableau. La diffusion collective empêche notamment les retours en arrière et les arrêts sur image, que chacun ferait individuellement.

Les résultats obtenus à partir des décomptes des durées consacrées aux différentes activités vont à l'encontre de nos attentes, au point que l'écoute apparaît même significativement plus importante $(U(16,48)=1,68 ; p<$ .10) dans le cours en amphithéâtre ( $c f$. Tableau 5). Contrairement aux déclarations des apprenants, le décrochage n'est pas significativement différent entre les deux situations $(U(18,46)=1,47 ;$ n.s.). La prise de notes et l'observation des supports ne diffèrent pas non plus significativement selon les conditions de cours $(U(25,39)=$ $0,73$; n.s. et $U(28,36)=0,42 ;$ n.s. $)$.

Tableau 5. Durées moyennes en secondes (sur un total observé de 600) des activités observables en situations d'enseignement classique et vidéodifférée

\begin{tabular}{lcccccc}
\hline \multirow{2}{*}{ Situations } & \multicolumn{6}{c}{ Comportements des apprenants (Durée en s) } \\
& $\begin{array}{l}\text { Prise de } \\
\text { notes }\end{array}$ & Écoute & Observ. & Décroch. & $\begin{array}{c}\text { Prise } \\
\text { d'info. }\end{array}$ & $\begin{array}{c}\text { Restitution } \\
\text { d'info. }\end{array}$ \\
\hline Classique & 360,2 & 98,5 & 108,4 & 32,9 & 206,9 & 360,2 \\
Vidéodifférée & 396,2 & 57,6 & 129,1 & 18,1 & 186,7 & 396,2 \\
\hline
\end{tabular}

Le fait qu'il n'y ait qu'un seul comportement observable qui fasse l'objet d'un contraste en les deux situations laisse penser que l'autonomie des apprenants est très peu influencée par le mode de diffusion d'un même cours.

\section{Discussion}

Dans la première étude, nous avons observé des variations significatives de la structuration des propos de l'enseignant ainsi que des variations significatives du comportement des apprenants. Ces variations sont conformes à la théorie de Moore, selon laquelle des cours de structure différente renverraient à une DT différente, qui se manifeste par une autonomie des apprenants différente, elle aussi. En contrôlant les conditions de structure du cours, la deuxième étude nous a permis de mieux observer la relation entre la distance et le dialogue. Il ressort que la mise à distance d'un cours selon des moyens synchrones, entraînant ainsi une supposée augmentation de la DT, ne s'accompagne pas d'une variabilité des paramètres observés. Cela peut signifier que les conditions de dialogue de ces deux situations sont trop proches l'une de l'autre et ne créent donc pas de variabilité suffisante; à moins que le caractère universitaire du cours (privilégiant le contenu du cours au dialogue) diminue la variabilité du dialogue. Enfin, au cours de la troisième étude, c'est l'effet de la distance sur l'autonomie des apprenants qui est questionnée, à la suite du contrôle de la structure et du dialogue. L'autonomie semble être indépendante de la distance, puisqu'il y a très peu de variation du comportement entre les deux situations.

Quoiqu'il en soit, les résultats que nous avons obtenus ne sont pas surprenants : lorsqu'on augmente le contrôle des variables indépendantes en jeu dans une expérimentation, il est normal que les différences entre les variables dépendantes s'estompent. Ainsi, nous sommes passés d'une comparaison de deux situations 
écologiques donnant de nombreuses différences significatives, tant au niveau du discours de l'enseignant que du comportement des apprenants; à deux comparaisons plus contrôlées de deux situations, dans lesquelles seul le discours de l'enseignant ou seul le comportement des apprenants pouvaient varier, ce qui, dans les deux cas ne s'est pas avéré.

Pour revenir à la notion de DT, il faut rappeler que nos observations ne portent pas sur tout le spectre des situations qu'elle peut couvrir. En effet, nous n'avons étudié que des enseignements en présence et à distance dispensés de façon synchrone. Si les résultats présentés ici tendent à montrer que la DT n'est pas aussi variable que son auteur la présente, nous ne pouvons le soutenir que dans des circonstances synchrones. D'autres travaux sont nécessaires, notamment pour mettre cette notion à l'épreuve dans des circonstances asynchrones bien contrôlées.

Par ailleurs, indépendamment de nos résultats, nous avons montré l'intérêt de méthodes fines d'analyse du discours de l'enseignant et du comportement de l'apprenant en situation d'EAD synchrone. Souvent employées dans des études sur l'EAD asynchrone (e.g., Baker et al., 2001), de telles méthodes d'analyse ont également une utilité dans des observations plus macroscopiques, comme celles présentées supra. Toutefois, il reste à réaliser d'autres études de ce type, afin d'essayer de mettre au jour des différences significatives sur des variables, plus fines que celles recueillies ici, liées notamment au discours de l'enseignant ou au comportement des apprenants.

\section{Remerciements}

Nous remercions vivement Pierre Bailly, qui s'est prêté de bonne grâce à nos observations des deux premières expérimentations et Yvan Abernot, dont le cours a été l'objet d'étude de la troisième expérimentation. Nous remercions également Jacques Baillé et Benoît Lemaire pour leur participation aux articles des deux premières expérimentations. Nous n'oublions pas non plus Odile Girod et Frédérique Tognarelli pour leur assistance dans l'analyse morphosyntaxique des données de la deuxième expérimentation, ainsi que Laurent Herzog pour sa contribution à l'analyse des comportements dans la troisième étude.

\section{Références}

[BAK 01] Baker, M., de Vries, E., Lund, K, \& Quignard, M. «Interactions épistémiques médiatisées par ordinateur pour 1'apprentissage des sciences : bilan de recherches », Sciences et Techniques Éducatives, 8(1-2), 2001, 21-32, accédé le 21 janvier 2003 à http://www.loria.fr/ quignard/publis/EIAO01.pdf

[BOU 00] Bouchard, P. "Autonomie et distance transactionnelle dans la formation à distance», In S. Alava (Ed.), Cyberespace et formations ouvertes, Bruxelles, De Boeck, 2000, pp. 65-78.

[BRO 85] Bronckart, J.-P. Le fonctionnement des discours, Neuchâtel, Delachaux et Niestlé, 1985.

[BRU 91] Bru, M. Les variations didactiques dans l'organisation des conditions d'apprentissage, Toulouse, Editions Universitaires du Sud, 1991.

[CAV 01] Cavanaugh, C. S. «The effectiveness of interactive distance education technologies in K-12 learning: a metaanalysis ", International Journal of Educational Telecommunications, 7(1), 2001, 73-88, Accédé le 10 janvier 2003 à http://www.unf.edu/ ccavanau/CavanaughIJET01.pdf

[CHE 98] Chen, Y.-J. « A path analysis of the concepts in Moore's theory of transactional distance in a videoconferencing learning environment», Journal of Distance Education, 13(2), 1998, Accédé le 27 juin 2003 à http://cade.athabascau.ca/vol13.2/chen.html

[CLA 83] Clark, R. E. « Reconsidering research on learning from media », Review of Educational Research, 53(4), 1983, 445-459.

[CLA 94] Clark, R. E. « Media will never influence learning », Educational Technology Research and Development, 42(2), 1994, 21-29.

[DES 97] Dessus, P., Lemaire, B., \& Baillé, J. «Études expérimentales sur l'enseignement à distance », Sciences et Techniques Éducatives, 4(2), 1997, 137-164.

[DES 99] Dessus, P., \& Lemaire, B. «La technologie des médias joue-t-elle un rôle dans la transmission des connaissances?», In S. Agostinelli (Ed.), Comment penser la communication des connaissances ? Du CD-Rom à l'Internet, Paris, L'Harmattan, 1999, pp. 253-264, Accédé le 10 janvier 2003 à http://www.upmfgrenoble.fr/sciedu/pdessus/media99.PDF

[DES 03] Desmoulins, C., Marquet, P., \& Bouhineau, D. (Éds.). Actes de la conférences EIAH 2003 (Environnements Informatiques pour l'Apprentissage Humain), Paris, INRP, 2003.

[DES soumis] Dessus, P. «Les effets de la distance sur le contenu d'un cours : une analyse avec LSA », soumis à publication.

[DRI 99] Dringus, L. P., \& Terrell, S. «The framework for DIRECTED online learning environments », The Internet and Higher Education, 2(1), 1999, 55-67.

[EHR 95] Ehrmann, S. C. " Asking the right question. What does research tell us about technology and higher learning?», Change Magazine, 27(2), 1995, 20-27, accédé le 5 mars 2002 à www.learner.org/edtech/rscheval/rightquestion.html 
[GAG 82] Gagné, R. M., \& Reiser, R. A. « Characteristics of media selection models », Review of Educational Research, 52(4), 1982, 499-512.

[GAR 00] Garrison, R. «Theoretical challenges for distance education in the 21st century: A shift from structural to transactional issues », International Review of Research in Open and Distance Learning, 1(1), 2000, accédé le 10 janvier 2003 à http://www.irrodl.org/content/v1.1/randy.pdf

[JUN 01] Jung, I. « Building a theoretical framework of web-based instruction in the context of distance education », British Journal of Educational Technology, 2001, accédé le 27 juin 2003 à http://home.ewha.ac.kr/ isjung/data/BJET-WBI.pdf

[KOZ 91] Kozma, R. B. « Learning with media », Review of Educational Research, 61(2), 1991, 179-211.

[KOZ 94] Kozma, R. B. « Will media influence learning? Reframing the debate », Educational Technology Research and Development, 42(2), 1994, 7-19.

[KUL 89] Kulik, J. A., \& Kulik, C.-L. C. « Meta-analysis in education », International Journal of Educational Research, 13(3), 1989, 223-340.

[LAN 97] Landauer, T. K., \& Dumais, S. T. «A solution to Plato's problem : the Latent Semantic Analysis theory of acquisition, induction and representation of knowledge », Psychological Review, 104, 1997, 211-240, accédé le 10 janvier 2003 à http://sa.colorado.edu/papers/plato/plato.annote.html

[LEM 97] Lemaire, B., Marquet, P., \& Baillé, J. «Étude comparative du discours d'un enseignant et du comportement des enseignants en situation de cours traditionnel et d'enseignement à distance informatisé », Carrefour de l'Éducation, 3, 1997, 76-91.

[LEM 98] Lemaire, B., Dessus, P., \& Baillé, J. « The Teacher Discourse at a Distance : Lexical, Morphosyntactical and Pragmatic Aspects », International Journal of Educational Telecommunications, 4(4), 1998, 367-381, accédé le 10 janvier 2003 à http://www.upmf-grenoble.fr/sciedu/pdessus/ijet98.PDF

[LEM 03] Lemaire, B., \& Dessus, P. « Modèles cognitifs issus de l'Analyse de la sémantique latente », Cahiers Romans de Sciences Cognitives, 1(1), 2003, 55-74, accédé le 27 juin 2003 à http://www.upmfgrenoble.fr/sciedu/pdessus/crsc03.PDF

[LOC 99] Lockee, B. B., Burton, J. K., \& Cross, L. H. « No comparison: Distance education finds a new use for 'No Significant Difference' », Educational Technology Research and Development, 47(3), 1999, 33-42.

[LOC 01] Lockee, B., Moore, M., \& Burton, J. « Old concerns with new distance education research », Educause Quarterly, 2, 2001, 60-62, accédé le 10 janvier 2003 à http://www.educause.edu/ir/library/pdf/eqm0126.pdf

[MAC 00] Machtmes, K., \& Asher, J. W. « A meta-analysis of the effectiveness of telecourses in distance education », The American Journal of Distance Education, 14(1), 2000, 27-46.

[MAR 99] Marquet, P., \& Herzog, L. «Le comportement d'auditeur en situation d'enseignement vidéo-différé et ses conséquences sur la vidéo synchrone ou asynchrone en ligne ou hors ligne », In P. Marquet, S. Mathey, A. Jaillet, \& E. Nissen (Eds.), Internet-Based Teaching and Learning (IN-TELE 98), Francfort, Lang, 1999, pp. 425-430.

[MOO 93] Moore, M. G. "Theory of transactional distance», In D. Keegan (Ed.), Theoretical principles of distance education, New York, Routledge, 1993, pp. 22-38.

[PER 92] Périn, P., \& Gensolen, M. (Eds.) La communication plurielle, l'interaction dans les télécommunications, Paris, La Documentation Française, 1992.

[PER 00] Peraya, D. «Le cyberespace : un dispositif de communication et de formation médiatisées », In S. Alava (Ed.), Cyberespace et formations ouvertes, Bruxelles, De Boeck, 2000, pp. 17-44.

[PHI 99] Phipps, R., \& Merisotis, J. What's the difference? A review of contemporary research on the effectiveness of distance learning in higher education, Washington, The Institute for Higher Education Policy, 1999, accédé le 10 janvier 2003 à http://www.ihep.com/Pubs/PDF/Difference.pdf

[ROM 92] Romiszowski, A. J. The Selection and Use of Instructional Media, Londres, Kogan Page, 1992.

[RUS 02] Russell, T. L. "The 'No significant difference' phenomenon », Raleigh, Université de Caroline du Nord, bureau des télécommunications éducatives, 2002, site Internet accédé le 30 juin 2003 à http://teleeducation.nb.ca/nosignificantdifference/

[SAB 00] Saba, F. «Research in Distance Education : A status report», International Review of Research in Open and Distance Learning, 1(1), 2000, 1-9, accédé le 10 janvier 2003 à http://www.irrodl.org/content/v1.1/farhad.pdf

[SAL 77] Salomon, G., \& Clark, R. E. « Reexamining the methodology of research on media and technology in education », Review of Educational Research, 47(1), 1977, 99-120.

[TEP 98] Teplovs, C., \& van Aalst, J. «Latent Semantic Analysis of CSILE/KF Databases », Proc. Annual Meeting and Conference of Telelearning NCE' '98, Vancouver.

[VAN 02] van Bruggen, J. M., Kirschner, P. A., \& Jochems, W. « External representation of argumentation in CSCL and the management of cognitive load», Learning and Instruction, 12(1), 2002, 121-138, accédé le 10 janvier 2003 à http://www.ou.nl/info-alg-english-r_d/OTEC_research/publications/artikelen/External_Representation_of_ Argumentation in_CSCL_Learning_Instruction.pdf

[WIS 01] Wisher, R. A., \& Olson, T. M. "The effectiveness of online instruction : Practices from the evaluation literature », Communication au Symposium "Assessing the quality of online instruction”, Monterey, Naval Postgraduate School, 2001, accédé le 10 janvier 2003 à http://ocl.nps.navy.mil/Conferences/archives/aqoi/briefs/WisherMonterey.ppt

[WOL 98] Wolfe, M. B. W., Schreiner, M. E., Rehder, B., Laham, D., Foltz, P., Kintsch, W., \& Landauer, T. K. « Learning from text: Matching readers and texts by Latent Semantic Analysis », Discourse Processes, 25(2-3), 1998, 309-336, accédé le 21 janvier 2003 à http://lsa.colorado.edu/papers/dp2.wolfe.pdf 